\title{
Correlation between obstructive sleep apnea hypopnea syndrome and hypertension: a systematic review and meta-analysis
}

\author{
Fengjuan Yuan $^{1,2}$, Shanshan Zhang ${ }^{1,2}$, Xiao Liu ${ }^{1,2}$, Yali Liu ${ }^{1,2}$ \\ ${ }^{1}$ Pingdingshan University Medical College, Pingdingshan, China; ${ }^{2}$ Philippine Women's University School of Nursing, Manila, Philippines \\ Contributions: (I) Conception and design: F Yuan, Y Liu; (II) Administrative support: S Zhang; (III) Provision of study materials or patients: S Zhang, \\ X Liu, Y Liu; (IV) Collection and assembly of data: All authors; (V) Data analysis and interpretation: F Yuan, X Liu, Y Liu; (VI) Manuscript writing: \\ All authors; (VII) Final approval of manuscript: All authors. \\ Correspondence to: Yali Liu. Pingdingshan University Medical College, Chongwen Road, Xinhua District, Pingdingshan, China. \\ Email: liuyali15037556689@163.com.
}

\begin{abstract}
Background: Obstructive sleep apnea hypopnea syndrome (OSAHS) is defined as more than 30 recurrent episodes of apnea or a sleep apnea hypopnea index (AHI) of more than 5 times/hour at a night. It is more common in people over 40 years old and is more prevalent in men. The pathogenesis factors and correlations of OSAHS are worth studying.

Methods: A literature search was performed in the PubMed, Embase, and Ovid-Medline databases from the date when the database was established to April 2021. Keywords included obstructive sleep apnea hypopnea syndrome, sleep apnea hypopnea syndrome, hypertension, and correlation.

Results: Eight articles involving 3,484 OSAHS patients were included. Analysis results showed that OSAHS was correlated with hypertension [odds ratio $(\mathrm{OR})=6.44,95 \%$ confidence interval (CI): 5.38-7.71, $\mathrm{P}<0.00001]$. The severity of OSAHS revealed that the correlation of hypertension to mild, moderate, and severe OSAHS showed OR $=1.93,95 \%$ CI: $0.69-5.43, \mathrm{P}=0.21, \mathrm{OR}=0.43,95 \% \mathrm{CI}: 0.24-0.74, \mathrm{P}=0.0003$, and $\mathrm{OR}=0.85,95 \% \mathrm{CI}: 0.42-1.73, \mathrm{P}=0.66$, respectively. The results indicated that mild, moderate, and severe OSAHS are risk factors for hypertension.

Discussion: Eight articles were included to determine the correlation between OSAHS and hypertension. It was found that OSAHS was closely correlated with hypertension, and they may be risk factors for each other.
\end{abstract}

Keywords: Obstructive sleep apnea hypopnea syndrome (OSAHS); correlation; meta-analysis; hypertension

Submitted Sep 27, 2021. Accepted for publication Dec 08, 2021.

doi: 10.21037/apm-21-3302

View this article at: https://dx.doi.org/10.21037/apm-21-3302

\section{Introduction}

Obstructive sleep apnea-hypopnea syndrome (SAHS) (OSAHS) refers to symptoms such as hypoventilation and apnea due to collapse and obstruction of upper airway, accompanied by snoring, sleep structure disorders, and other phenomenon. Apnea refers to the cessation of oral and nasal airflow during sleep for $\geq 10 \mathrm{~s}$. Hypoventilation is defined as the intensity of respiratory airflow is $30 \%$ or more lower than the basic level during the sleep, accompanied by blood oxygen saturation $(\mathrm{SaO}) 3 \%$ or arousal. OSAHS is one of the most common sleep disordered respiratory illnesses in the
United States. Recurrent hypoxemia, hypercapnia, and sleep structure disorders at night lead to daytime sleepiness, cardiocerebral pulmonary vascular complications, and damages of multiple organs, which greatly impacts the life and threatens the lives of patients (1). OSAHS is clearly associated with hypertension, and continuous positive airway pressure (CPAP) therapy is the first-line way to treat moderate to severe OSAHS (2). Previous studies on the antihypertensive effect of CPAP therapy in OSAHS complicated with hypertension have shown that multi-room and ambulate blood pressure were the endpoints, and home-based blood 
pressure measurement intervention based on the Internet of Things (IoT) technology could significantly lower the blood pressure (3). According to its pathogenesis, SAHS can be divided into OSAHS, central SAHS, and mixed SAHS, among which OSAHS is the most common (4). In recent years, OSAHS has been widely recognized as a systemic disease and an independent risk factor for many cardiovascular diseases, including hypertension, atherosclerosis, arrhythmia, and heart failure; in addition, it shows close correlation to the prognosis of cardiovascular diseases (5). Previous studies have found that OSAHS shows a clear correlation with hypertension. In 2008, the American Heart Association/American Cardiology Foundation (AHA/ ACCF) reported that approximately $50 \%$ of OSAHS patients suffer from hypertension, and at least one-third hypertensive patients are associated with OSAHS $(6,7)$.

Secondary hypertension is accompanied with definite etiology, and often manifests as refractory high blood pressure. When the cause is identified and effectively removed or controlled, hypertension can be cured or controlled (8). OSAHS and aortitis are two common causes of secondary hypertension (9), and OSAHS is one of the important causes of refractory hypertension. Many patients (no less than $30 \%$ ) with hypertension have OSAHS, and the incidence of hypertension in OSAHS is as high as $50-80 \%$ $(10,11)$. If OSAHS is not controlled, it is difficult for blood pressure to return to normal, especially at night and in the morning (12). Currently, CPAP is the first-line treatment for OSAHS, and previous studies have focused on its effects on in-room and ambulate blood pressure (13). The curative effect of CPAP therapy relies on the compliance of patients in long-term therapy. The so-called compliance refers to the proportion of time for patients to apply machine treatment according to the set pressure. This is influenced by many different factors, such as treatment equipment, the degree of patients' understanding of the disease, etc. (14). Some patients cannot tolerate or do not receive CPAP therapy, but OSAHS is difficult to control if not corrected (15).

However, there are relatively few clinical reports on the relationship between OSHAS and hypertension. The relationship between OSAHS and hypertension requires further exploration. Therefore, a systematic analysis was developed here to analyze the relationship between these two conditions, in order to provide an important theoretical basis and support for the subsequent treatment of secondary hypertension. We present the following article in accordance with the MOOSE reporting checklist (available at https://dx.doi.org/10.21037/apm-21-3302).

\section{Methods}

\section{How to search the articles}

The comprehensive and systematic literature review in this paper was performed by referring to the Cochrane Handbook of Systematic reviews of interventions, with metaanalyses reported according to the PRISMA and MOOSE (Meta-analysis of Observational Studies in Epidemiology) guidelines. The PubMed, scientific conferences, and established articles were searched. All of the studies included patients treated for migraine. Specifically, all articles regarding the relationship between OSAHS and hypertension were searched using keywords and medical titles containing the following: "obstructive sleep apnea hypopnea syndrome", "high blood pressure", etc.

\section{How to determine the qualified articles}

Articles which met the following conditions were included: (I) study type: prospective cohort study or casecontrol study; (II) subjects: OSAHS patients diagnosed by polysomnography and non-OSAHS patients; (III) outcome indicators: the correlation between OSAHS and hypertension; and (IV) the experimental data were not missing, the sample size was exact, and the intergroup data of the study object was complete.

\section{Diagnostic criteria of OSAHS}

The criteria of adult OSAHS diagnosis and treatment guidelines (draft) formulated by the Sleep Respiratory Disease Group, Respiratory Society of Chinese Medical Association in 2002 were adopted, which included patients with $>30$ recurrent episodes of apnea and hypopnea during 7 hours of sleep breathing per night, or a sleep apnea hypopnea index $(\mathrm{AHI}) \geq$ five times/h (16). According to the AHI values, OSAHS patients were graded into mild (AHI 5-20 times/h), moderate (AHI 21-40 times/h), and severe (AHI >40 times/h).

\section{Diagnostic criteria for hypertension}

The diagnostic criteria for hypertension published by the Chinese Hypertension Prevention and Control Guidelines Revision Committee [known as the Chinese Guidelines for Hypertension Prevention and Control (2005 revision)] were adopted. This included patients with systolic blood pressure (SBP) no lower than $140 \mathrm{mmHg}$ and diastolic blood pressure (DBP) no lower than $90 \mathrm{mmHg}$ without antihypertensive drugs. Patients with a previous history of hypertension who were currently taking antihypertensive 
medications were diagnosed with hypertension even though their blood pressure was $<140 / 90 \mathrm{mmHg}$ (17).

Articles which met following conditions had to be excluded: (I) the literatures published for many times due to different evaluation indexes in the same clinical trial were excluded, and cases where the extract the first published data; (II) literature that is inconsistent with the evaluation indexes of this study or does not provide valid data; (III) low-quality literature; (IV) Repeatedly included literature; (V) articles with unclear diagnostic criteria or incorrect data analysis methods, studies that did not provide the OR (risk ratio, RR) value and $95 \%$ confidence interval (CI), and articles that included data that could be converted into a OR (RR) value and 95\% CI.

\section{Literature screening}

The two researchers first screened the titles and abstracts independently according to the qualified criteria, and then extracted and evaluated the quality of the data. When the evaluation results were inconsistent, other researchers were consulted to resolve the discrepancy according to the original data. If the titles and abstracts could meet the given requirements, it could retrieve the full text and perform the data extraction. Note express 2.0 was used for literature management and duplicate literature was deleted. The literature was checked strictly according to the inclusion and exclusion criteria mentioned above and relevant literature was traced back.

\section{Data extraction}

The two researchers extracted relevant information independently, and a predefined data extraction table was adopted. The information to be extracted included the author, year of publication, sample size, age, country, gender, degree of disease, and course of disease. The diagnostic criteria for ulcerative colitis (UC) and colorectal cancer (CRC) were as follows: if any data could not be found in the original article, it could try to contact the authors via E-mail to get the information. For data that was not available, the Cochrane evaluation manual was used for relevant transformation.

\section{Quality assessment}

To improve the quality of reviewed literature, their quality was evaluated with the "risk of bias assessment" recommended the Cochrane system review manual (version 5.3). The evaluation included the following seven items: (I) randomization method; (II) allocation concealment; (III) implementation of a blinding method between patients and researchers; (IV) evaluation of the effect of the blinding method; (V) Other biases for the RCTs in Item 7 above, "satisfied" means that the bias is relatively small. "Dissatisfied" means that the bias is high. The study does not have sufficient detailed reports. If not mentioned, the risk is unknown. The evaluation includes four dimensions: random sequence generation, allocation, concealment, blind method and tracking/exit. A score of 1-3 in the four dimensions of tracking/exit was considered low quality, and a score of 4-7 was considered high quality.

\section{Data analysis}

The forest plot also clearly shows the results of individual studies, combining those studies with corresponding CIs. If the Cis ware not overlapped, there is statistical inhomogeneity among different articles. In this case, it has to conduct the subgroup analysis to combine stochastic and fixed models with acceptable inhomogeneity. Inhomogeneity between the studies could not be ignored when different properties were investigated, and different properties cannot be addressed with in order to deal with the inhomogeneity. Select combined statistical model.

\section{Sensitivity analysis}

Sensitivity was performed on the research results by checking the degree of overall results to be affected by individual articles. Each article was taken out one at a time. The combined results of each study were analyzed to confirm the consistence degree of the results. Generally, this study believes that it will have an impact on the comprehensive study when the below two conditions are satisfied. Firstly, if a study is deleted, the size presumption of the combined effect reaches $95 \%$, and the results are great different. Moreover, if any article can cause little change of overall results, it means the sensitivity is unstable; or otherwise, the sensitivity is stable and the conclusion is correct.

\section{Statistical analysis}

Review Manager 5.3 was used for data processing in this systematic review, and the test level was 0.05 . $\mathrm{I}^{2}<50 \%$ and $\mathrm{P}>0.05$ indicated no statistical heterogeneity among trials, and then the meta-analysis was realized using fixed effects model (FEM). However, $\mathrm{I}^{2} \geq 50 \%$ and $\mathrm{P} \leq 0.05$ indicated 


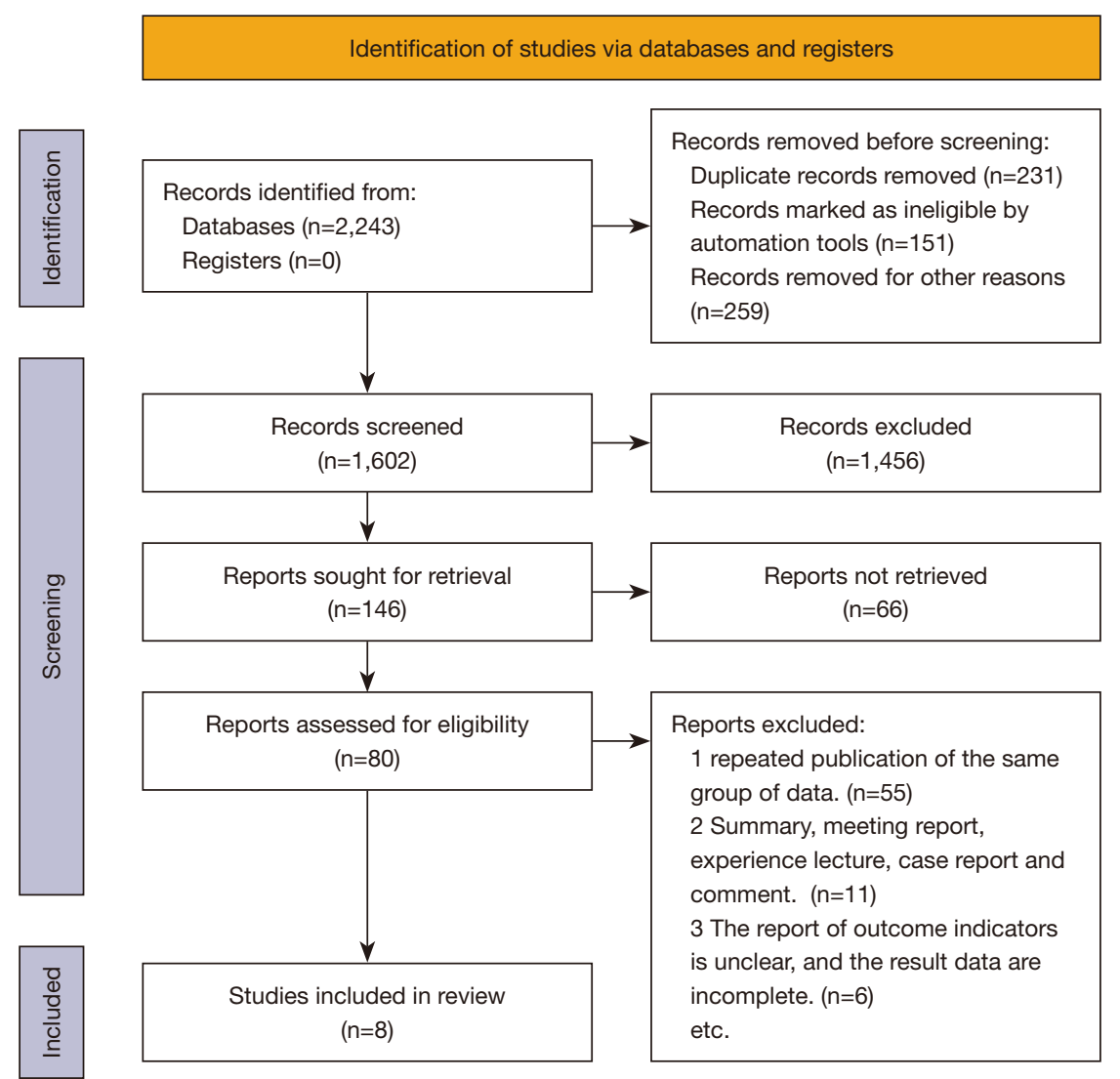

Figure 1 Literature retrieval process.

statistical heterogeneity between the trials, and random effects model (REM) was selected for meta-analysis. The combined effect size of the evaluation index data of both groups was the OR value and its $95 \% \mathrm{CI}$, and a forest map was drawn according to the integrated system evaluation results to display the research conclusions. Results with a high heterogeneity were analyzed using piecewise exclusion to explore the possible sources of heterogeneity, and sensitivity on the results was analyzed accordingly. If the number of randomized controlled trial (RCT) of an indicator is $\geq 5$, publication bias should be evaluated and a funnel plot should be used.

\section{Results}

\section{Literature search results}

In this study, a total of 2,243 relevant literatures were initially retrieved, including 1,084 from PubMed, 498 from Embase, 303 from Ovid-Medline, and 358 in other databases. According to the standard, 641 articles were initially excluded; 1,456 articles were excluded after the titles and abstracts were read. After reading the full texts of the remaining articles, a further 66 articles were deleted. After carefully reading the full text of the literature, 72 articles were excluded, and eight studies (18-25) that met the inclusion criteria were finally included (Figure 1, Table 1).

\section{Bias-risk assessment}

The risk of bias of the articles were evaluated using Cochrane Handbook (version 5.0.2), and the corresponding charts were drawn by Review Manager 5.3 (Figures 2,3).

Newcastle-Ottawa Scale (NOS) scores were adopted to evaluate the quality of each article. The results revealed that the eight articles all have a low risk of bias, which satisfied the requirements of subsequent analysis (Table 2).

\section{Meta-analysis results}

No statistical heterogeneity was found among the eight 
Table 1 Basic information of articles for analysis

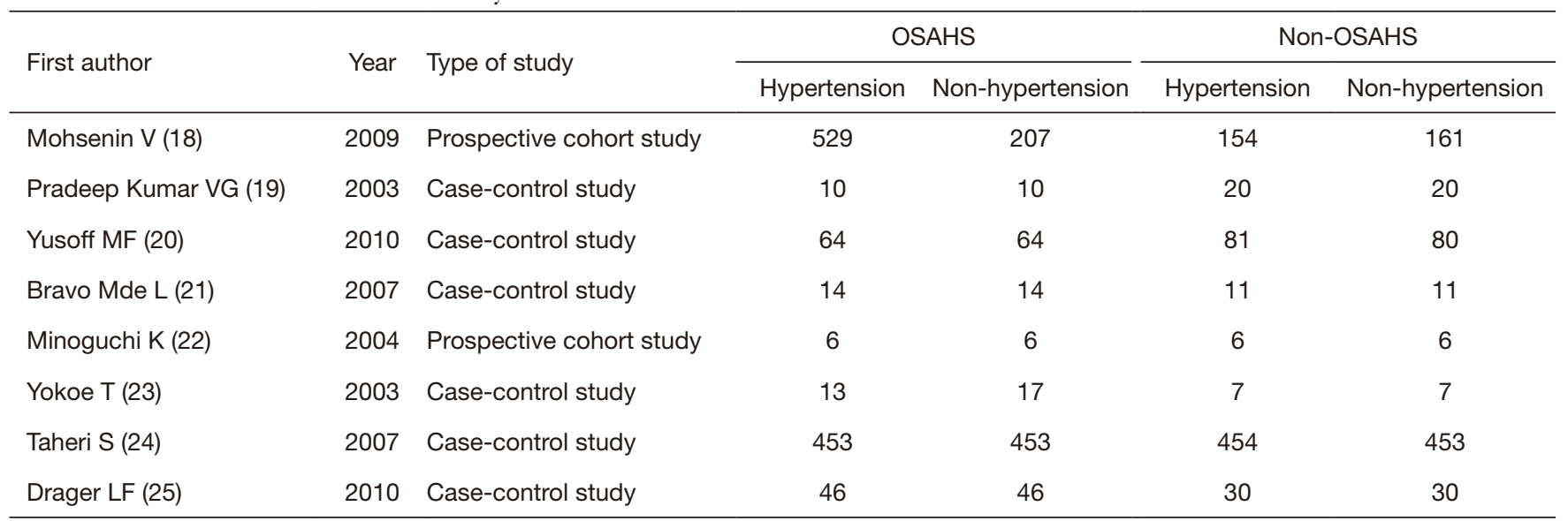

OSAHS, obstructive sleep apnea hypopnea syndrome.

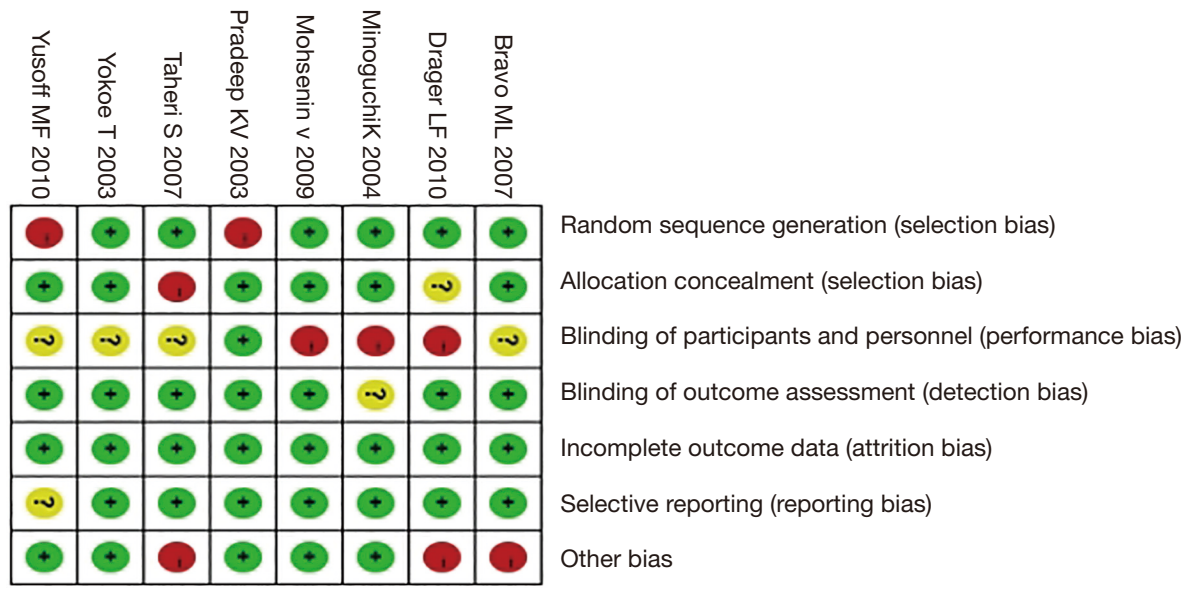

Figure 2 The assessment results of risk of bias.

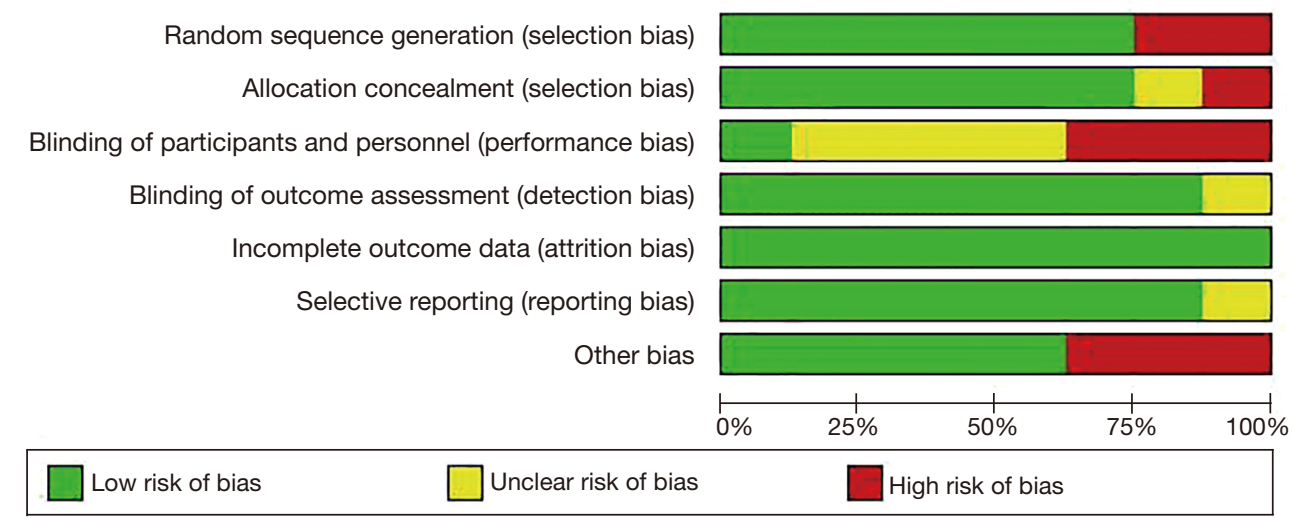

Figure 3 The bias evaluation results. 
Table 2 NOS scores of the articles

\begin{tabular}{|c|c|c|c|c|c|c|c|c|c|c|c|}
\hline First author & Year & 1 & 2 & 3 & 4 & $5 A$ & $5 B$ & 6 & 7 & 8 & Total points \\
\hline Pradeep Kumar VG (19) & 2003 & Yes & No & No & Yes & Yes & Yes & Yes & Yes & Yes & 7 \\
\hline Yusoff MF (20) & 2010 & Yes & No & No & Yes & Yes & No & Yes & Yes & Yes & 6 \\
\hline Bravo Mde L (21) & 2007 & Yes & No & No & Yes & Yes & No & Yes & Yes & Yes & 6 \\
\hline Yokoe T (23) & 2003 & Yes & No & Yes & Yes & Yes & No & Yes & Yes & Yes & 7 \\
\hline Taheri S (24) & 2007 & Yes & No & Yes & No & Yes & Yes & Yes & Yes & Yes & 7 \\
\hline Drager LF (25) & 2010 & Yes & No & Yes & Yes & Yes & No & Yes & Yes & Yes & 7 \\
\hline
\end{tabular}

NOS, Newcastle-Ottawa Scale.

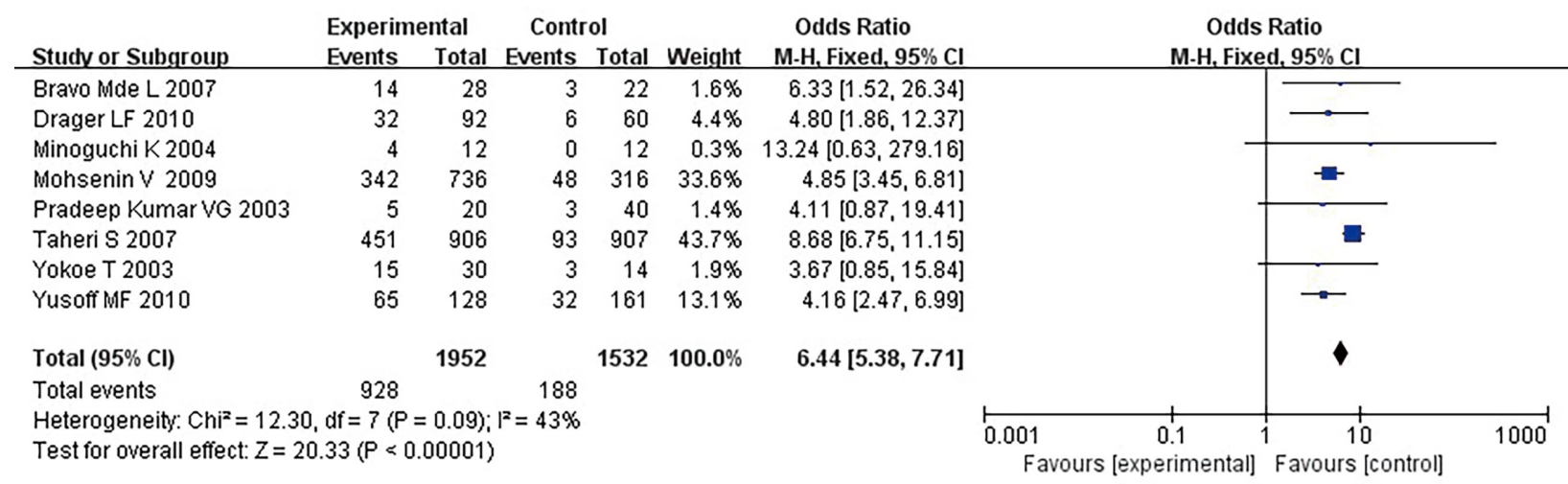

Figure 4 Correlation between OSAHS and hypertension. OSAHS, obstructive sleep apnea hypopnea syndrome.

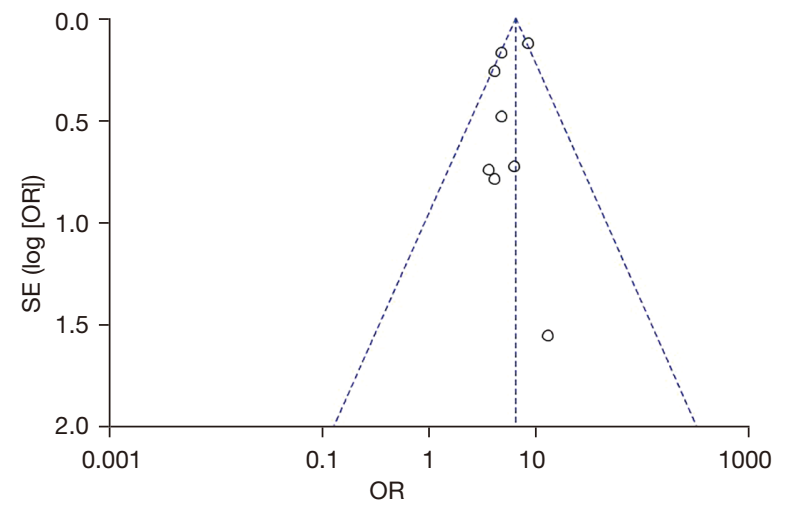

Figure 5 Funnel plot analysis of the correlation between OSAHS and hypertension. OSAHS, obstructive sleep apnea hypopnea syndrome. articles for meta-analysis $\left(\mathrm{P}=0.09, \mathrm{I}^{2}=43 \%\right)$. A FEM was selected for pooled analysis. A statistically obvious difference could be visible between the two groups (OR =6.44, 95\% CI: 5.38-7.71, $\mathrm{P}<0.00001$ ), suggesting that OSAHS was correlated with hypertension (Figure 4).

Funnel plot analysis on the association between OSAHS and hypertension of the eight included studies was conducted, and the results showed that the funnel plot was asymmetric, which suggested that the publication bias is possible (Figure 5).

\section{Correlation between OSAHS and bypertension in different types of studies}

The correlation between OSAHS and hypertension in 


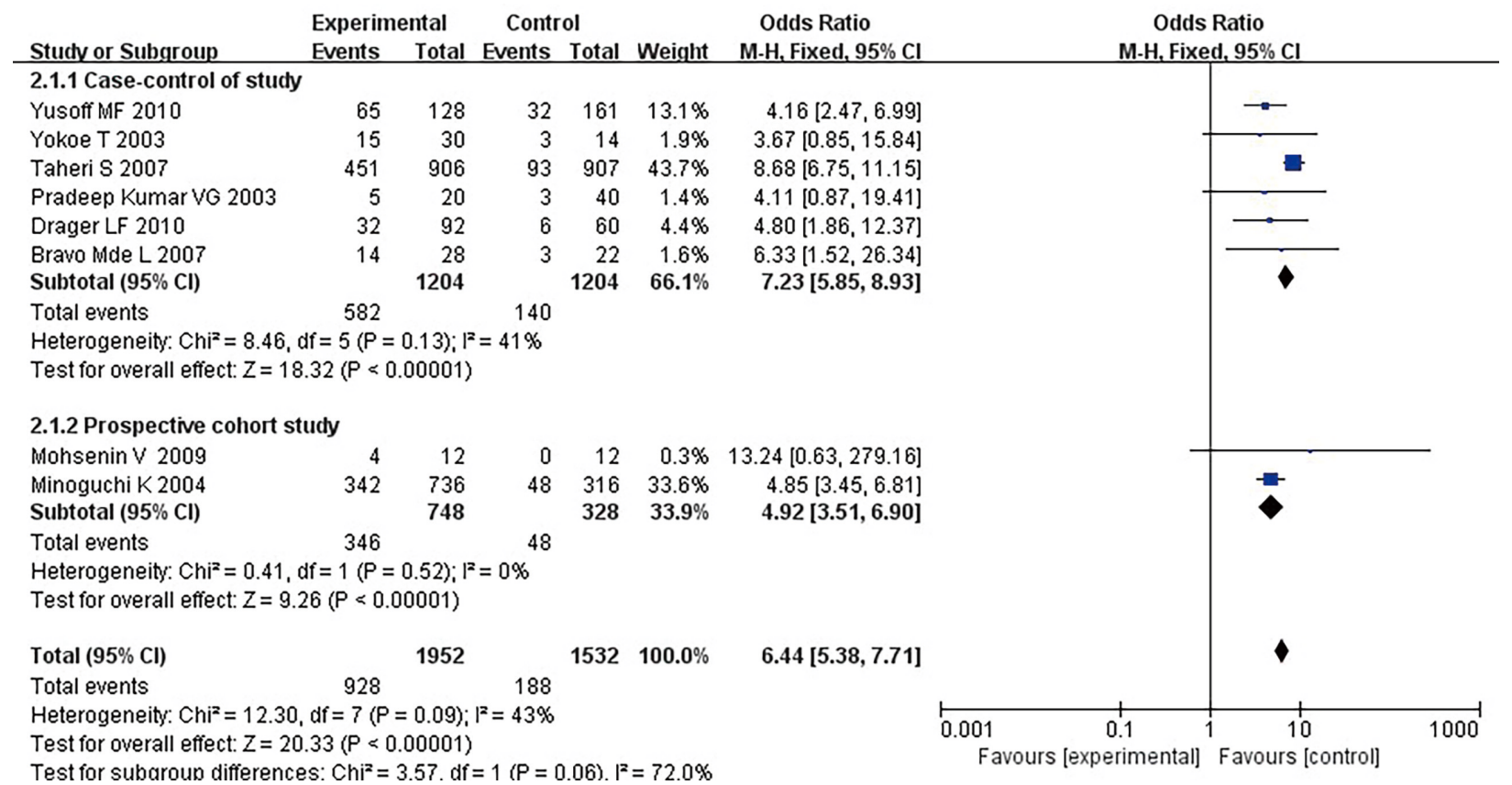

Figure 6 Meta-analysis of the association between OSAHS and hypertension (subgroup by study type). OSAHS, obstructive sleep apnea hypopnea syndrome.

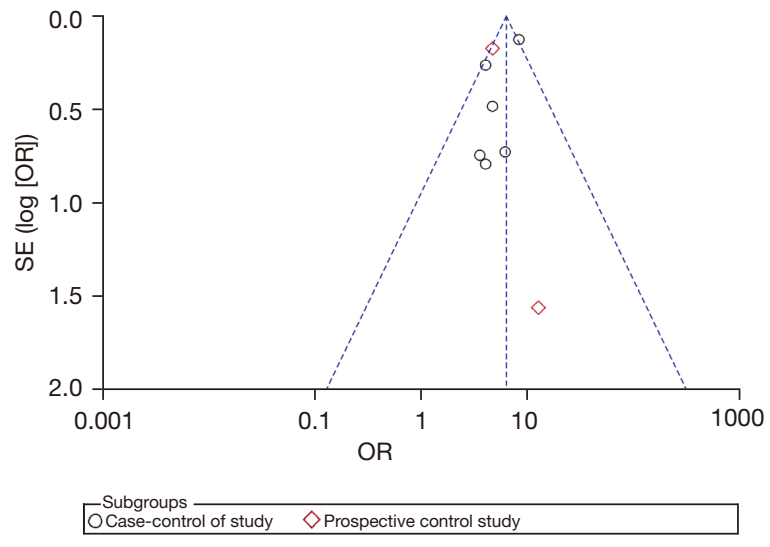

Figure 7 Funnel plot analysis of the correlation between OSAHS and hypertension (subgroup by study type). OSAHS, obstructive sleep apnea hypopnea syndrome.

different study types was divided into two subgroups according to the different study types of the included articles. It was found no statistical heterogeneity among the six articles in the case-control subgroup $\left(\mathrm{P}=0.13, \mathrm{I}^{2}=41 \%\right)$. The FEM analysis results showed that a statistically observably difference could be found (OR $=7.23,95 \%$ CI: 5.85-8.93, $\mathrm{P}<0.00001$ ), suggesting that OSAHS was correlated with hypertension. The subgroup of the cohort study showed that the two groups were not greatly and statistically different (OR $=6.44$, 95\% CI: 5.38-7.71, $\mathrm{P}=0.06$ ), suggesting no correlation between them (Figure 6).

Funnel plot analysis on the correlation between OSAHS and hypertension was conducted for studies of different types (Figure 7).

\section{Correlation between OSAHS of different severity and bypertension}

The correlation between OSAHS of different severity and hypertension was analyzed in the eight included studies. Three studies (two case-control studies and one prospective cohort study) grouped OSAHS patients according to the severity of the disease and observed the incidence of hypertension. The patients were divided into three subgroups for meta-analysis: mild, moderate, and severe cases. The results were as follows.

In the subgroup of patients with mild OSAHS, no statistical heterogeneity was found $\left(\mathrm{P}=0.08, \mathrm{I}^{2}=60 \%\right)$, so the pooled analysis was realized using FEM. The difference was visible statistically ( $\mathrm{OR}=1.93,95 \%$ CI: $0.69-5.43$, $\mathrm{P}=0.21$ ), suggesting that mild OSAHS was correlated with 


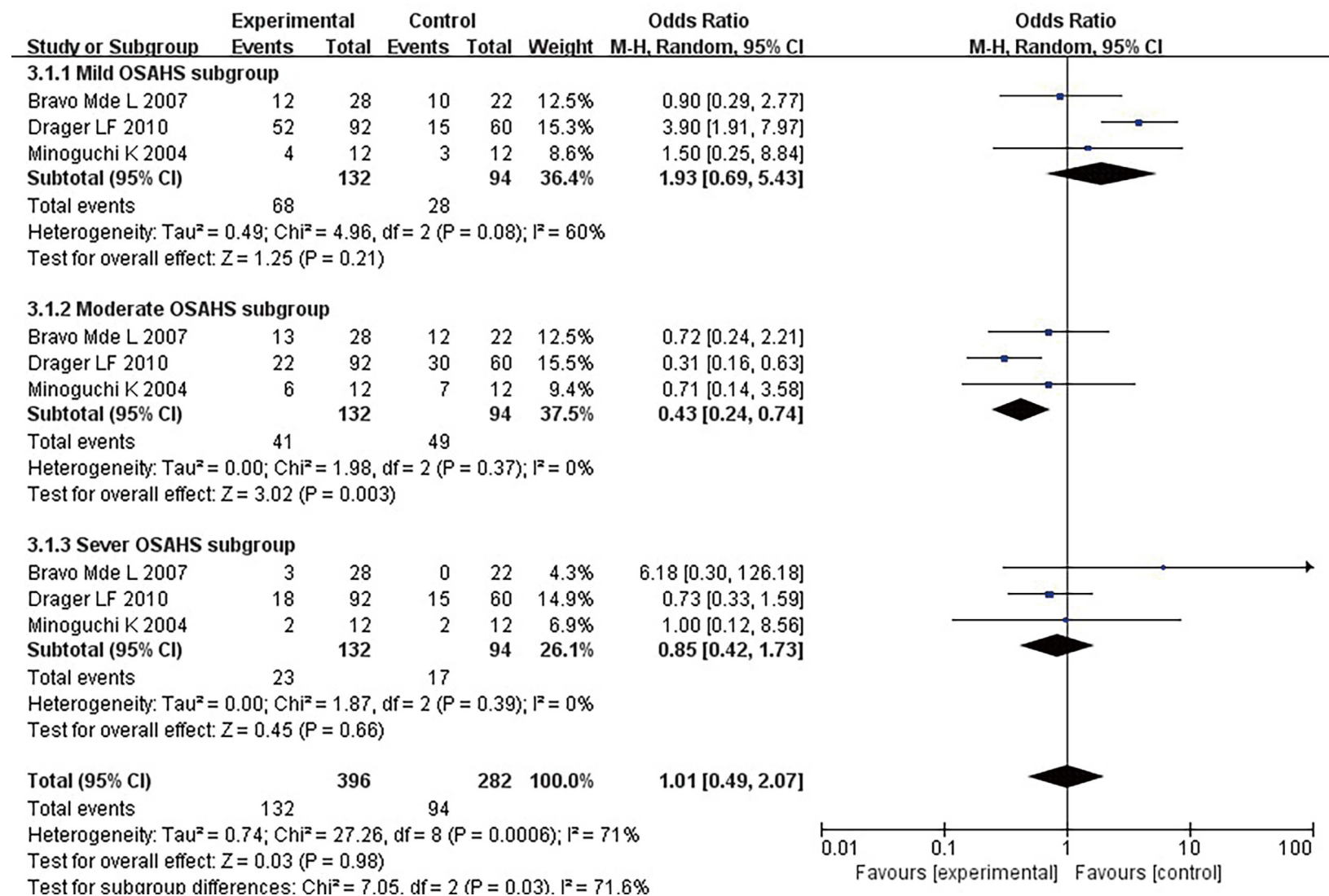

Figure 8 Correlation between OSAHS and hypertension (subgroups according to the severity of OSAHS). OSAHS, obstructive sleep apnea hypopnea syndrome.

hypertension.

In the subgroup of patients with moderate OSAHS, statistical heterogeneity was not found $\left(\mathrm{P}=0.37, \mathrm{I}^{2}=0 \%\right)$, so the FEM was adopted. The difference was proved to be statistically great for patients in different groups $(\mathrm{OR}=0.43$, 95\% CI: 0.24-0.74, $\mathrm{P}=0.003$ ), suggesting that mild OSAHS was correlated with hypertension.

The articles showed no statistical heterogeneity for patients with severe OSAHS ( $\left.\mathrm{P}=0.39, \mathrm{I}^{2}=0 \%\right)$, so the FEM was determined for pooled analysis. The results suggested that the difference was statistically remarkable $(\mathrm{OR}=0.85$, 95\% CI: 0.42-1.73, $\mathrm{P}=0.66$ ), suggesting that severe OSAHS was correlated with hypertension.

The above results show that OSAH is correlated with hypertension regardless of severity (Figure 8).

Funnel plot analysis on the correlation between OSAHS of different severity and hypertension was conducted, and the results are shown in Figure 9.

\section{Discussion}

OSAHS is a common sleep apnea disorder with high incidence and considerable harm, which lowers the quality of life of patients and even threatens their lives greatly. Numerous studies have found that OSAHS may be a risk factor for various cardiovascular diseases, and approximately $30-50 \%$ of OSAHS patients suffer from hypertension (26). Numerous foreign scholars (27) have proposed that OSAHS patients suffer from recurrent episodes of insufficient ventilation or even apnea during sleep, leading to intermittent hypoxemia, hypercapnia, and $\mathrm{pH}$ depensation, which all stimulate chemoreceptors in the central and cardiovascular centers, resulting in increased blood pressure at night and disruption of the circadian rhythm of blood pressure. According to relevant reports (28), the ability of OSAHS patients to regulate the pressure reflex is reduced, and their blood pressure increases repeatedly, eventually leading to persistent hypertension. Some studies have found 


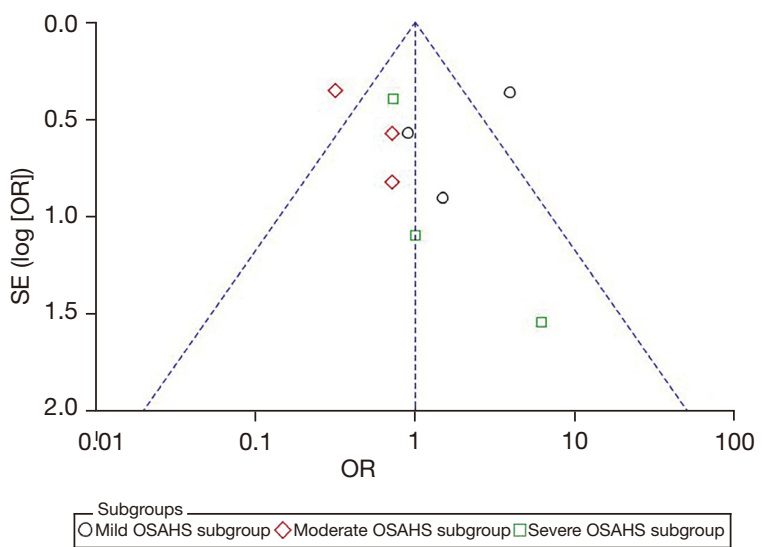

Figure 9 Funnel plot analysis of the correlation between OSAHS and hypertension (subgroups according to the severity of OSAHS). OSAHS, obstructive sleep apnea hypopnea syndrome.

that the change of blood pressure is related to sleep apnea, and relevant clinical experiments suggest that the longer the hypoxia caused by OSAHS, the higher the risk coefficient of hypertension, and the two are linearly correlated (29). In addition, obesity and other factors may also be risk factors leading to the increased incidence of hypertension in OSAHS patients (30).

Regarding the prevention of OSAHS, first avoid your own obesity; try to lie on your side when you sleep at night to reduce the supine position; quit smoking and drinking; avoid bad lifestyles such as staying up late and tired. And control high-salt and high-oil diets, quit smoking, limit alcohol intake, keep exercising, be careful not to stay up late, and avoid excessive stress. These are all measures to prevent high blood pressure. The results of this systematic evaluation show that OSAHS is correlated with hypertension, and the incidence of hypertension in OSAHS patients is higher in contrast to that in the control group, suggesting that OSAHS is an independent risk factor for hypertension. Furthermore, we found that OSAHS was correlated with hypertension regardless of its severity.

\section{Conclusions}

This article embarks from the high blood pressure, to the pathogenesis of OSAHS is studied. This research studies the pathogenesis of OSAHS from hypertension. In this paper, a total of eight articles were analyzed, and the results showed that high blood pressure is closely and positively related to the occurrence of OSAHS. However, due to the limited search conditions, some studies may be missed. Also, the quality of the most of the included studies was not high, which may have resulted in further limitations.

\section{Acknowledgments}

Funding: None.

\section{Footnote}

Reporting Checklist: The authors have completed the MOOSE reporting checklist. Available at https://dx.doi. org/10.21037/apm-21-3302

Conflicts of Interest: All authors have completed the ICMJE uniform disclosure form (available at https://dx.doi. org/10.21037/apm-21-3302). The authors have no conflicts of interest to declare.

Ethical Statement: The authors are accountable for all aspects of the work in ensuring that questions related to the accuracy or integrity of any part of the work are appropriately investigated and resolved.

Open Access Statement: This is an Open Access article distributed in accordance with the Creative Commons Attribution-NonCommercial-NoDerivs 4.0 International License (CC BY-NC-ND 4.0), which permits the noncommercial replication and distribution of the article with the strict proviso that no changes or edits are made and the original work is properly cited (including links to both the formal publication through the relevant DOI and the license). See: https://creativecommons.org/licenses/by-nc$\mathrm{nd} / 4.0 /$.

\section{References}

1. Wu ZH, Yang XP, Niu X, et al. The relationship between obstructive sleep apnea hypopnea syndrome and gastroesophageal reflux disease: a meta-analysis. Sleep Breath 2019;23:389-97.

2. Otto-Yáñez M, Torres-Castro R, Nieto-Pino J, et al. Obstructive sleep apnea-hypopnea and stroke. Medicina (B Aires) 2018;78:427-35. 
3. Ko CY, Liu QQ, Su HZ, et al. Gut microbiota in obstructive sleep apnea-hypopnea syndrome: diseaserelated dysbiosis and metabolic comorbidities. Clin Sci (Lond) 2019;133:905-17.

4. D'Cruz RF, Murphy PB, Kaltsakas G. Sleep disordered breathing and chronic obstructive pulmonary disease: a narrative review on classification, pathophysiology and clinical out-comes. J Thorac Dis 2020;12:S202-16.

5. Pérez-Carbonell L, Bashir S. Narrative review of sleep and stroke. J Thorac Dis 2020;12:S176-90.

6. Lerousseau L. Sleepiness, continuous positive airway pressure and the obstructive sleep apnea hypopnea syndrome. Rev Mal Respir 2018;35:116-33.

7. Chen JN, Liu YL, Lu MZ, et al. Immunological mechanism of tumorigenesis promoted by obstructive sleep apnea hypopnea syndrome. Zhonghua Jie $\mathrm{He} \mathrm{He} \mathrm{Hu}$ Xi Za Zhi 2019;42:609-12.

8. Rimoldi SF, Scherrer U, Messerli FH. Secondary arterial hypertension: when, who, and how to screen? Eur Heart J 2014;35:1245-54.

9. Tziomalos K. Secondary Hypertension: Novel Insights. Curr Hypertens Rev 2020;16:11.

10. Charles L, Triscott J, Dobbs B. Secondary Hypertension: Discovering the Underlying Cause. Am Fam Physician 2017;96:453-61.

11. Rossi GP, Bisogni V, Rossitto G, et al. Practice Recommendations for Diagnosis and Treatment of the Most Common Forms of Secondary Hypertension. High Blood Press Cardiovasc Prev 2020;27:547-60.

12. Maspero C, Giannini L, Galbiati G, et al. Obstructive sleep apnea syndrome: a literature review. Minerva Stomatol 2015;64:97-109.

13. Gupta S, Donn SM. Continuous positive airway pressure: Physiology and comparison of devices. Semin Fetal Neonatal Med 2016;21:204-11.

14. Villanueva JA, Isetta V, Montserrat JM, et al. A Portable Continuous Positive Airway Pressure Device That Can Perform Optimally under Strenuous Conditions. Am J Respir Crit Care Med 2018;198:956-8.

15. Campos-Rodriguez F, Navarro-Soriano C, Reyes-Nuñez $\mathrm{N}$, et al. Good long-term adherence to continuous positive airway pressure therapy in patients with resistant hypertension and sleep apnea. J Sleep Res 2019;28:e12805.

16. Morley C, Davis P. Continuous positive airway pressure: current controversies. Curr Opin Pediatr 2004;16:141-5.

17. Mireles-Cabodevila E, Kacmarek RM. Should Airway
Pressure Release Ventilation Be the Primary Mode in ARDS? Respir Care 2016;61:761-73.

18. Mohsenin V, Yaggi HK, Shah N, et al. The effect of gender on the prevalence of hypertension in obstructive sleep apnea. Sleep Med 2009;10:759-62.

19. Pradeep Kumar VG, Bhatia M, Tripathi M, et al. Obstructive sleep apnoea: a case-control study. Neurol India 2003;51:497-9.

20. Yusoff MF, Baki MM, Mohamed N, et al. Obstructive sleep apnea among express bus drivers in Malaysia: important indicators for screening. Traffic Inj Prev 2010;11:594-9.

21. Bravo Mde L, Serpero LD, Barceló A, et al. Inflammatory proteins in patients with obstructive sleep apnea with and without daytime sleepiness. Sleep Breath 2007;11:177-85.

22. Minoguchi K, Tazaki T, Yokoe T, et al. Elevated production of tumor necrosis factor-alpha by monocytes in patients with obstructive sleep apnea syndrome. Chest 2004;126:1473-9.

23. Yokoe T, Minoguchi K, Matsuo H, et al. Elevated levels of C-reactive protein and interleukin-6 in patients with obstructive sleep apnea syndrome are decreased by nasal continuous positive airway pressure. Circulation 2003;107:1129-34.

24. Taheri S, Austin D, Lin L, et al. Correlates of serum C-reactive protein (CRP)--no association with sleep duration or sleep disordered breathing. Sleep 2007;30:991-6.

25. Drager LF, Lopes HF, Maki-Nunes C, et al. The impact of obstructive sleep apnea on metabolic and inflammatory markers in consecutive patients with metabolic syndrome. PLoS One 2010;5:e12065.

26. Pérez-Rico C, Gutiérrez-Díaz E, Mencía-Gutiérrez E, et al. Obstructive sleep apnea-hypopnea syndrome (OSAHS) and glaucomatous optic neuropathy. Graefes Arch Clin Exp Ophthalmol 2014;252:1345-57.

27. Sun WT, Chen Y, Lu HH, et al. An analysis of electrocochleography in patients with OSAHS. Lin Chung Er Bi Yan Hou Tou Jing Wai Ke Za Zhi 2018;32:697-9.

28. Peng BG, Lai YQ, Lei HJ, et al. Strategies in the clinical diagnosis and surgical treatment of OSAHS with multilevel obstruction. J Int Med Res 2019;47:1533-43.

29. Rong T, Ma JG, Zhao YL, et al. The evaluation of severity and treatment for OSAHS by acoustic pharyngometry 
which in different postures. Lin Chung Er Bi Yan Hou Tou Jing Wai Ke Za Zhi 2019;33:118-22.

30. Lu X, Wang $\mathrm{X}, \mathrm{Xu} \mathrm{T}$, et al. Circulating $\mathrm{C} 3$ and glucose metabolism abnormalities in patients with OSAHS. Sleep

Cite this article as: Yuan F, Zhang S, Liu X, Liu Y. Correlation between obstructive sleep apnea hypopnea syndrome and hypertension: a systematic review and meta-analysis. Ann Palliat Med 2021;10(12):12251-12261. doi: 10.21037/apm-21-3302
Breath 2018;22:345-51.

(English Language Editor: A. Kassem) 